\title{
Circular RNA hsa_circ_0000326 acts as a miR-338-3p sponge to facilitate lung adenocarcinoma progression
}

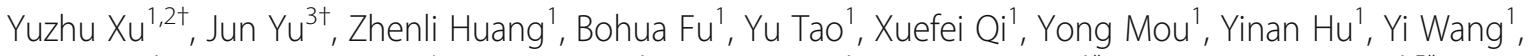
Yong $\mathrm{CaO}^{1}$, Dingsheng Jiang ${ }^{4}$, Jungang $\mathrm{Xie}^{1}$, Yongjian $\mathrm{Xu}^{1}$, Jianping Zhao ${ }^{1 *}$ and Weining Xiong ${ }^{1,5^{*}}$

\begin{abstract}
Background: Circular RNAs (circRNAs) are a novel class of noncoding RNAs that regulate gene expression at the transcriptional or posttranscriptional level. According to recent studies, circRNAs are involved in the pathogenesis of cancer, but the roles of circRNAs in lung adenocarcinoma are largely unknown.

Methods: In this study, we identified a novel upregulated circRNA, hsa_circ_0000326, in human lung adenocarcinoma tissues using microarray analysis and qRT-PCR. We then explored the biological role of hsa_circ_0000326 using gainand loss-of-function assays in adenocarcinoma cells. Bioinformatics databases were used to screen for potential target miRNAs and the luciferase reporter assays and RNA-FISH further validated the interaction. Downstream protein was detected by western blot. Finally, we established xenografts in nude mice to assess the function of hsa_circ_0000326 in vivo.

Results: We found that high expression of hsa_circ_0000326 was correlated with tumor size, regional lymph node status and differentiation in human lung adenocarcinoma. Additionally, we conducted gain- and loss-of-function assays and found that hsa_circ_0000326 acted as a positive regulator of cell proliferation and migration and a negative regulator of apoptosis. Mechanistic studies showed that hsa_circ_0000326 acted as a miR-338-3p sponge and altered the function of miR-338-3p, which in turn upregulated the expression of the downstream target RAB14 and affected the proliferation, migration and apoptosis of lung adenocarcinoma cells.

Conclusions: Collectively, our study results reveal crucial roles for hsa_circ_0000326 in the proliferation, migration and apoptosis of lung adenocarcinoma cells and suggest that hsa_circ_0000326 may represent a potential therapeutic target in patients with lung adenocarcinoma.
\end{abstract}

\footnotetext{
* Correspondence: zhaojp@tjh.tjmu.edu.cn; xiongdoctor@qq.com

${ }^{\dagger}$ Yuzhu Xu and Jun Yu contributed equally to this work.

'Department of Respiratory and Critical Care Medicine, Wuhan Clinical Medical Research Center for Chronic Airway Medicine, NHC Key Laboratory of Pulmonary Diseases, Key cite of National Clinical Research Center for Respiratory Diseases, Tongji Hospital, Tongji Medical College, Huazhong University of Sciences \& Technology, 1095 Jiefang Ave, Wuhan 430030, China Full list of author information is available at the end of the article
} 


\section{Introduction}

Lung cancer is one of the most common causes of cancer-related death [1], and the 5-year survival rate for patients with lung cancer is only $16.8 \%$ [2]. Non-small cell lung cancers (NSCLCs) account for $85 \%$ of all cases [3], among which lung adenocarcinoma is the most common histological type. Therefore, molecular studies aimed at identifying promising targets for the treatment of lung adenocarcinoma are urgently needed.

Circular RNAs (circRNAs) are produced by the circularization of exons, exons and introns, or intron sequences alone and are widely expressed in various cell types $[4,5]$. Since circRNAs usually do not display protein-coding potential, they were once considered simply to be accidental byproducts of splicing [6]. Advances in RNA sequencing technologies have accelerated the identification of circRNAs. CircRNAs are abundant and conserved RNA isoforms that are more stable than linear RNAs [7, 8]. Divergent primers (primers in a primer pair that face away from each other) that cross over back-splicing sites have been used to amplify particular circular transcripts [9]. CircRNAs have also been recognized as miRNA sponges and transcriptional regulators [9-11]. Moreover, they have been found to interact with RNA-binding proteins or be translated into proteins in vitro [12-14]. Further studies on circRNAs might provide new insights that will improve our understanding of pathological mechanisms and improve the prevention and diagnosis of related diseases. Previous studies have shown that circRNAs might be involved in the pathogenesis of lung cancer $[15,16]$. However, the exact roles of circRNAs in lung adenocarcinoma are far from known.

Hsa circ 0000326 is located on chromosome 11: $65272490-65,272,586$, and its associated gene symbol is MALAT1. Interestingly, in our early screening experiment, hsa_circ_0000326 was found to be markedly upregulated in a cohort of lung adenocarcinoma tissues and adjacent tissues by microarray analysis. Herein, we provide evidence that aberrant hsa_circ_0000326 expression could promote proliferation and migration and inhibit apoptosis in lung adenocarcinoma cells. Further study showed that hsa_circ_0000326 acted as a miR338-3p sponge to inhibit its activity and thus upregulate its target RAB14, which in turn affected proliferation, migration and apoptosis in lung adenocarcinoma cells. Based on these results from the present study, targeting hsa_circ_0000326 may be a viable treatment strategy for lung adenocarcinoma.

\section{Materials and methods}

\section{Ethics statement and patient samples}

Human lung adenocarcinoma tissues and corresponding adjacent tissues ( $>5 \mathrm{~cm}$ from the tumor edge) $(N=100)$ were obtained from patients who received surgical treatment at Tongji Hospital (Wuhan, China) from June 2014 to February 2015. Fresh tissues were immediately snap-frozen and stored at $-80^{\circ} \mathrm{C}$. All of the patients gave written informed consent, and none of the patients had previously undergone radiotherapy or chemotherapy. This study was approved by the Human Assurance Committee of Tongji Hospital, Tongji Medical College, Huazhong University of Science and Technology.

\section{CircRNA microarray}

Five pairs of lung adenocarcinoma and adjacent tissues were used to detect the expression of circRNAs with a CircRNA Microarray (KangChen Biotech, Shanghai, China). Sample preparation and microarray hybridization were performed according to standard protocols from Arraystar (Rockville, MD, USA). Briefly, total RNA from each sample was amplified and transcribed into fluorescent cRNA using random primers according to the Arraystar Super RNA Labeling Kit protocol. Labeled cRNAs were hybridized onto an Arraystar Human circRNA Array (Arraystar Inc., MD, USA). After the slides were washed, the arrays were scanned using an Axon GenePix 4000B microarray scanner. The scanned images were then imported into GenePix Pro 6.0 software for grid alignment and data extraction. Quantile normalization and subsequent data processing were performed using the $\mathrm{R}$ software package. All of the circRNAs array data were listed in Supplement Table 1.

\section{Cell culture}

Human lung adenocarcinoma cell lines (A549, SPC-A1, H1299 and H1975) were purchased from the Type Culture Collection of the Chinese Academy of Sciences (Shanghai, China). And the cell lines was authenticated by short tandem repeat method at Suzhou Genetic Testing Biotechnology Co.,Led. (Suzhou, China) in Feb., 2018. The cells were cultured in Roswell Park Memorial Institute (RPMI) 1640 medium supplemented with 10\% fetal bovine serum (FBS), $100 \mathrm{mg} / \mathrm{mL}$ streptomycin and $100 \mathrm{IU} / \mathrm{mL}$ penicillin in a $5 \% \mathrm{CO}_{2}$.

\section{Cell transfection}

Three siRNAs targeting hsa_circ_0000326 and a nontargeting control (named si-circ0000326-1, si-circ0000326-2, sicirc0000326-3 and si-NC, respectively), miRNA mimics, a miRNA inhibitor and a negative control miRNA (miRNANC) were obtained from RiboBio (Guangzhou, China). The sequence for siRNAs were as follows: si-circ0000326-1: $5^{\prime}$ GTAACTGGCATGTGAACAA-3'; si-circ0000326-2: 5' ACTGGCATGTGAACAAGCT-3'; si-circ0000326-3:5' TGGCATGTGAACAAGCTTT-3'. The sequence of mature hsa_circ_0000326 was synthesized and inserted into a circRNA vector plasmid (named pAV-circ0000326) from 
Vigene Biosciences (Rockville, MD, USA). The transfection of siRNAs and plasmids was optimized using Lipofectamine 3000 (Invitrogen, Carlsbad, CA, USA), according to the manufacturer's instructions.

\section{Generation of stable cell lines}

The si-circ0000326 or si-NC sequence was inserted into the lentiviral expression vector piLenti-siRNA-GFP and packaged into viral particles (LV-si-circ0000326 or LV$\mathrm{NC}$, respectively). H1299 cells were infected with either LV-si-circ0000326 or LV-NC particles. Three days after infection, the cells were selected in medium containing $5 \mu \mathrm{g} / \mathrm{mL}$ puromycin for 7 days and then maintained in medium containing $1 \mu \mathrm{g} / \mathrm{mL}$ puromycin.

\section{RNA extraction and RT-PCR}

Total RNA was isolated using TRIzol reagent (TaKaRa, Tokyo, Japan) as previously described $[17,18]$. For quantitative PCR analysis of circRNAs and mRNAs, total RNA (500 ng) from each sample was transcribed into first-strand cDNA using a Prime Script ${ }^{\mathrm{tm}}$ RT Master Mix kit (TaKaRa, Tokyo, Japan). Real-time PCR was performed using SYBR ${ }^{\circ}$ Premix Ex Taq ${ }^{\text {sim }}$ with an ABI Prism 7500 detection system according to the manufacturers' instructions. The data were analyzed using the $2^{-\Delta \Delta C t}$ method and normalized to $\beta$-actin expression levels. The primers for human hsa_circ_0000326 and $\beta$-actin were as follows: hsa_circ_0000326, forward: 5'-TTG AAT AGA TTT CAG CTT TAT GC-3' and reverse: $5^{\prime}$-CCC ATA ACT GAT CTG ACT TTG T-3'; $\beta$-actin, forward: 5'-CCT GGC ACC CAG CAC AAT-3' and reverse: 5'GGG CCG GAC TCG TCA TAC-3'.

To detect the miRNA expression level, a total of $1 \mu \mathrm{g}$ miRNAs were reverse transcripted with Bulge-LoopTM miRNA qRT-PCR Primer Set (Ribobio, Guangzhou, China) and the miR-338-3p expression was detected with SYBR ${ }^{\circ}$ Premix Ex Taq ${ }^{\text {mix }}$ (TaKaRa, Tokyo, Japan). Primers for miR-338-3p and U6 snRNA were purchased from Ribobio.

\section{Flow cytometry analysis}

For cell cycle assays, transfected cells were harvested, diluted to a density of $1 \times 10^{6}$ cells $/ \mathrm{mL}$ and fixed with $70 \%$ ice-cold ethanol. Next, the cells were stained with $400 \mu \mathrm{L}$ of a propidium iodide solution (Keygen Biotech, Nanjing, China) for $30 \mathrm{~min}$ and then subjected to cell cycle analysis using flow cytometry (BD Biosciences, San Jose, CA, USA).

For apoptosis analysis, $300 \mathrm{nM} \mathrm{H}_{2} \mathrm{O}_{2}$ was applied to induce apoptosis. Cells were collected $48 \mathrm{~h}$ after transfection and resuspended in binding buffer. The cells were then incubated with annexin $\mathrm{V}$ and propidium iodide (Keygen Biotech, Nanjing, China) for $15 \mathrm{~min}$ in the dark and then analyzed by flow cytometry on a FACSCalibur flow cytometer.

\section{Cell proliferation assay}

Transfected cells were seeded in a 96-well plate at a density of 4000 cells per well and cultured for $24 \mathrm{~h}, 48 \mathrm{~h}$, $72 \mathrm{~h}$ or $96 \mathrm{~h}$. Cell Counting Kit 8 (CCK-8) solution (Keygen Biotech, Nanjing, China) was added to each well, and the cells were then incubated at $37^{\circ} \mathrm{C}$ for $60 \mathrm{~min}$. The absorbance was measured at $450 \mathrm{~nm}$ with a spectrophotometer. The data are representative of three individual experiments performed in triplicate.

For colony formation assays, 450 transfected cells were seeded in each well of a 6-well plate and maintained for 10 days, with replacement of the medium every 3 days. The colonies were then fixed with methanol, stained with $0.5 \%$ crystal violet, and counted under a microscope.

\section{Transwell assay}

For the migration assay, $2 \times 10^{4}$ transfected cells were suspended in $200 \mu \mathrm{L}$ of serum-free medium and then seeded in the upper chamber of each transwell insert (Corning, NY, USA); $600 \mu \mathrm{L}$ of culture medium containing 15\% FBS was added to the lower chamber.

After $24 \mathrm{~h}$ of incubation, the cells that had migrated were fixed with $4 \%$ paraformaldehyde and stained with $0.5 \%$ crystal violet, and the cells remaining on the upper surface of the filter membrane were removed with a cotton swab. Images of cells on the lower surface of the membrane were captured using a microscope equipped with a camera.

\section{Western blot analysis}

Total proteins were extracted using RIPA lysis buffer supplemented with a protease inhibitor cocktail, as previously described $[19,20]$. Proteins were separated by SDS-PAGE and then blotted onto PVDF membranes. The membranes were incubated with primary antibodies overnight at $4{ }^{\circ} \mathrm{C}$ and then incubated with secondary antibodies for $1 \mathrm{~h}$ at room temperature. Finally, the proteins were detected by chemiluminescence according to the manufacturer's recommendations (Advansta, CA, USA). The following primary antibodies were used: antiRAB14 (1:1000, Proteintech, Wuhan, China), anti-ZEB2 (1:1000, Proteintech, Wuhan, China), anti-SOX4 (1:1000, Millipore, Billerica, MA, USA), and anti-GAPDH (1: 2000, Sungene, Tianjin, China).

\section{Bioinformatics analysis and luciferase reporter assay}

The relationships between circRNAs and miRNAs were predicted with custom Arraystar miRNA target prediction software based on miRanda (http://www.microrna.org) and TargetScan (www.targetscan.org). The target genes of miRNAs were acquired from TargetScan, PicTar and 
miRanda. The putative binding site of hsa_circ_0000326 or putative binding site mutants were inserted into the MCS of the pmirGLO vector for use in a dual luciferase reporter assay (abm, Richmond, Canada). HEK293T cells were cotransfected with wild-type or mutant pmirGLOhsa_circ_0000326 (WTpmirGLOcirc0000326 or MTpmirGLOcirc0000326, respectively) along with $50 \mathrm{nM}$ miRNC, miR-338-3p, miR-9-3p, miR-16-2-3p, miR-320a or miR-320b mimic using Lipofectamine 2000 (Life Technologies, MA, USA). Firefly and Renilla luciferase activity was measured with a Dual-Luciferase Reporter System (Promega, Madison, WI, USA) $48 \mathrm{~h}$ after transfection. The effect of each miRNA on the activity of the luciferase reporter containing the hsa_circ_0000326 sequence was normalized to the activity of the luciferase reporter cotransfected with miRNA-NC.

\section{Fluorescence in situ hybridization (FISH)}

RNA-FISH was used to detect the subcellular localization of hsa_circ_0000326 and miR-338-3p. Cells were grown on cover slips, fixed with $4 \%$ paraformaldehyde for 20 min, incubated with proteinase $\mathrm{K}$ and washed with a series of alcohol solutions. Then, the slides were washed and incubated with prehybridization solution (BersinBio, Guangzhou, China) for $30 \mathrm{~min}$ at $37^{\circ} \mathrm{C}$. FAM-labeled hsa circ_0000326 probes (5'-AAG CTT GTT CAC ATG CCA GTT ACT-3') and Cy3-labeled miR-338-3p probes (5'-CAA CAA AAT CAC TGA TGC TGG A-3') (Sangon Biotech, Shanghai, China) were denatured at $73^{\circ} \mathrm{C}$ for 8 min and hybridized to the slides for $24 \mathrm{~h}$ at $42^{\circ} \mathrm{C}$. Subsequently, blocking was performed, and 4,6-diamidino-2phenyl-indole (DAPI) was used to stain the cell nuclei. Images were obtained with a confocal microscope (Olympus, Shinjuku, Japan).

\section{RNA pulldown assay}

RNA pulldown assay was conducted by Shanghai Ruantuo Biological Technology Co. (Shanghai, China) using established techniques [21].

\section{Establishment of tumor xenografts in nude mice}

Male BALB/c nude mice (4-5 weeks of age) were purchased from Hunan SJA Laboratory Animal Company (Changsha, China) and maintained under specific pathogen-free conditions. Cells stably infected with LVsi-circ0000326 or LV-NC H1299 $\left(1 \times 10^{6}\right.$ cells in $100 \mu \mathrm{L}$ of PBS) were subcutaneously injected into the right armpits of nude mice. The tumor size was measured every 7 days. Three weeks later, the mice were sacrificed, and the weight of each tumor was measured.

\section{Statistical analysis}

All experiments were performed in triplicate. Continuous data are presented as the mean \pm standard deviation
(SD). Paired t tests, independent $\mathrm{t}$ tests, chi-square tests and one-way analysis of variance (ANOVA) were used in this study as appropriate. GraphPad Prism 5.0 software (GraphPad Software Inc., San Diego, CA, USA) and SPSS 22.0 software (IBM Corp., Armonk, NY, USA) were used for statistical analysis of the data. A two-tailed $P$-value $<0.05$ was considered to indicate a statistically significant difference.

\section{Results \\ Hsa_circ_0000326 expression was upregulated in lung adenocarcinoma tissues and associated with clinical characteristics}

A total of 8247 circRNAs were detected by microarray analysis in five pairs of lung adenocarcinoma and adjacent noncancerous tissues. Hierarchical clustering revealed that different circRNA expression patterns between cancer and adjacent noncancerous tissues (Fig. 1a). Scatter plots and volcano plots were used to visualize the differentially expressed circRNAs that showed statistically significant differences between tissue types (Fig. 1b and c). The expression of 568 circRNAs was altered in lung adenocarcinoma tissues, among which 448 circRNAs were upregulated and 120 circRNAs were downregulated (fold change $\geq 2.0, P<0.05$ and FDR $<0.05)$. The top ten upregulated and downregulated circRNAs are listed in Supplement Table 2. Consistent with the data from the microarray analysis, hsa circ_0000326 was found to be markedly upregulated in an expanded cohort of lung adenocarcinoma tissues and adjacent tissues $(N=100)$ by RT-PCR (Fig. 1d).

Next, correlations between hsa_circ_0000326 expression and patient clinical pathological parameters were analyzed, and the results revealed that hsa_circ_0000326 expression was significantly correlated with tumor size (T1 vs T3, $P=0.0143$; T2 vs T3, $P=0.0366$, Fig. 1 e), lymphatic metastasis $(P=0.004$, Fig. 1f) and tumor differentiation stage $(P<0.001$, Fig. $1 \mathrm{~g})$ in patients with lung adenocarcinoma. However, high expression of hsa circ_0000326 was not correlated with patient age $(P=$ $0.797)$, sex $(P=0.258)$, smoking status $(P=0.181)$ or distant metastasis $(P=0.537$, Fig. $2 \mathrm{~g})$ (Supplement Table 3). These results suggested a link between upregulation of hsa_circ_0000326 and aggressive characteristics of lung adenocarcinoma.

\section{Hsa_circ_0000326 promoted proliferation and migration and inhibited apoptosis in lung adenocarcinoma cells}

To further explore the role of hsa_circ_0000326 in the progression of lung adenocarcinoma, we performed lossof-function and gain-of-function assays. Based on the levels of endogenous hsa_circ_0000326 expression in the four lung adenocarcinoma cell lines (Fig. 1i), the A549 and H1299 cell lines were chosen as our experimental 


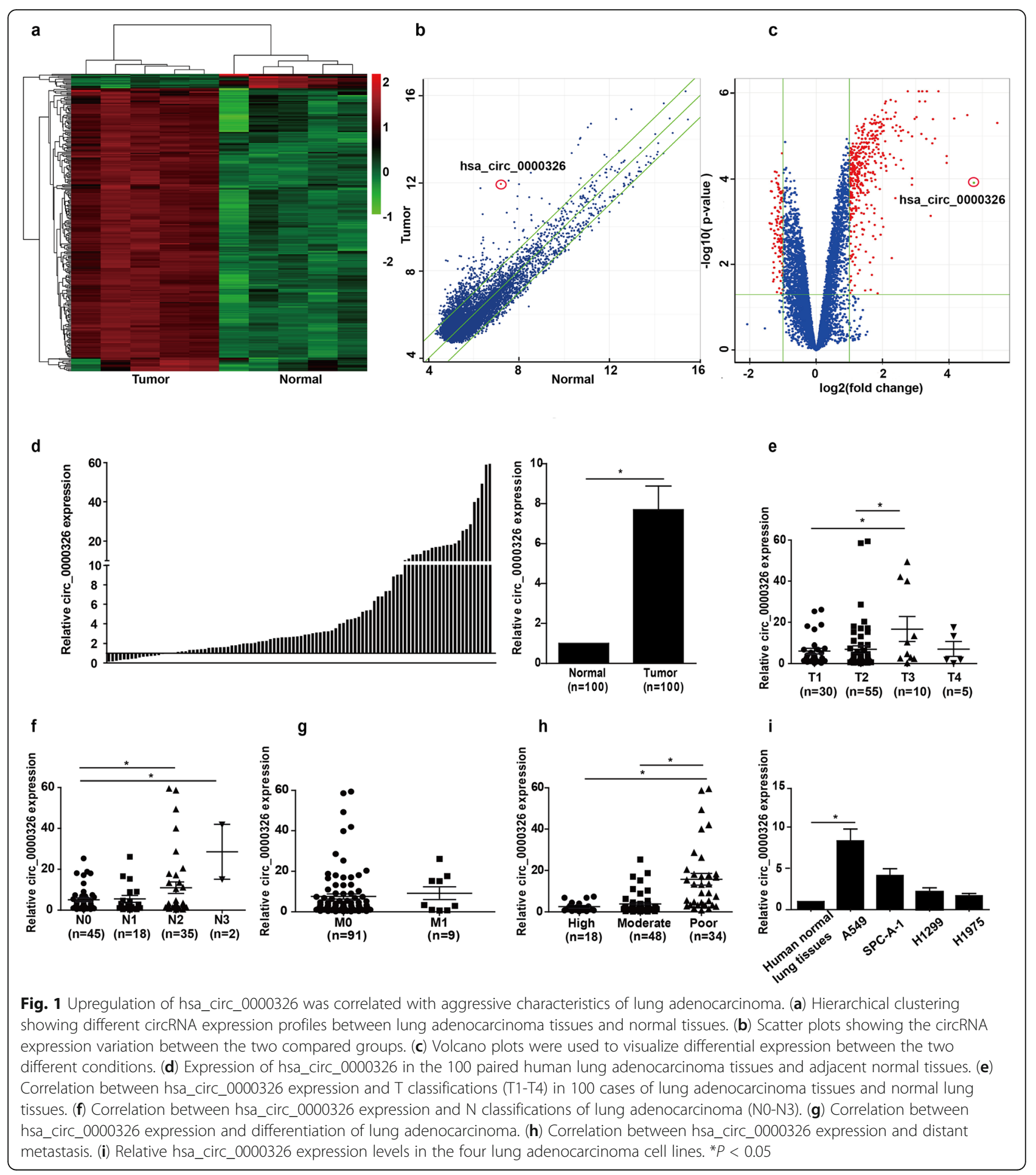

cell lines. Specifically, designed siRNAs targeting hsa circ_0000326 and si-NC were used to transfect cells. Hsa_circ_0000326 expression levels were reduced to the greatest extent in cells transfected with si-circ00003263 , which was thus used in subsequent analysis (Fig. 2a). In contrast, an hsa_circ_0000326 overexpression cell model was generated by transfecting an hsa_circ
0000326 expression plasmid into A549 or H1299 cells. RT-PCR was used to verify the upregulation of hsa_circ 0000326 in cells transfected with pAV-circ0000326 compared to those transfected with the negative control plasmid (pAV-NC) (Fig. 2f). Cell proliferation was assessed by CCK-8 assay. Silencing of hsa_circ_0000326 expression suppressed the proliferation of A549 and 


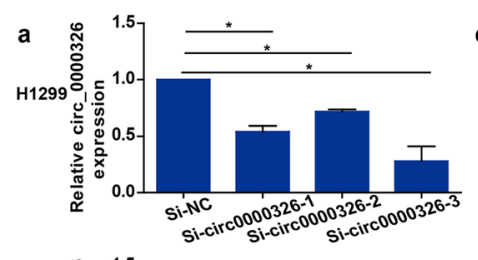

c
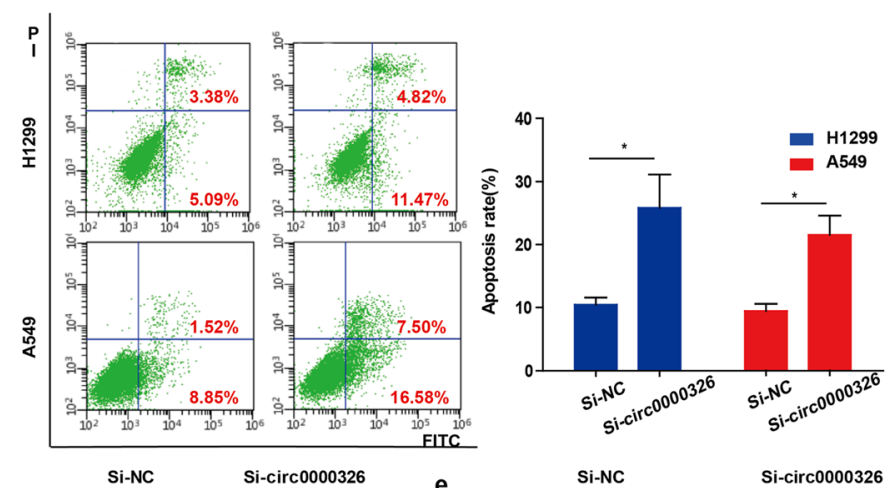

b
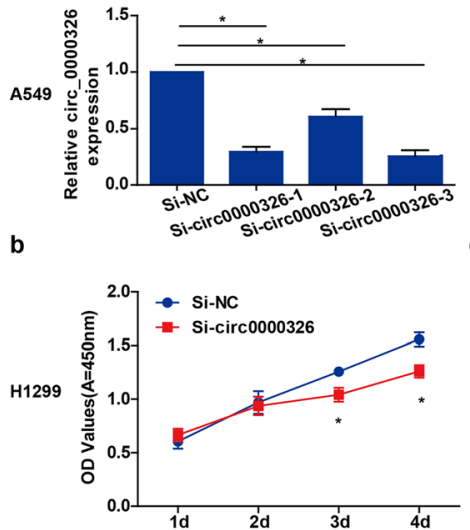

d
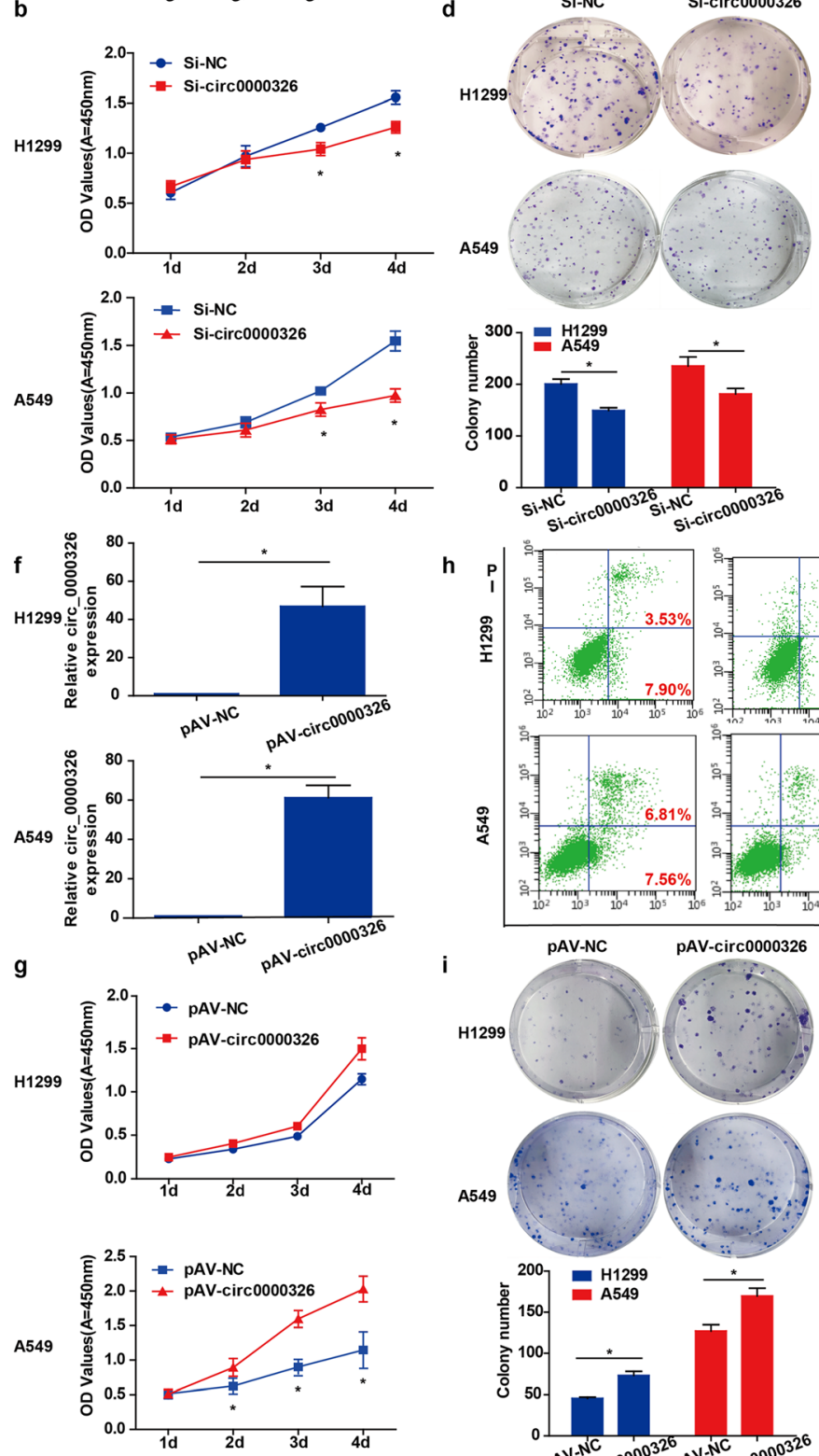

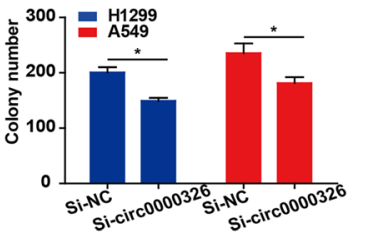

h
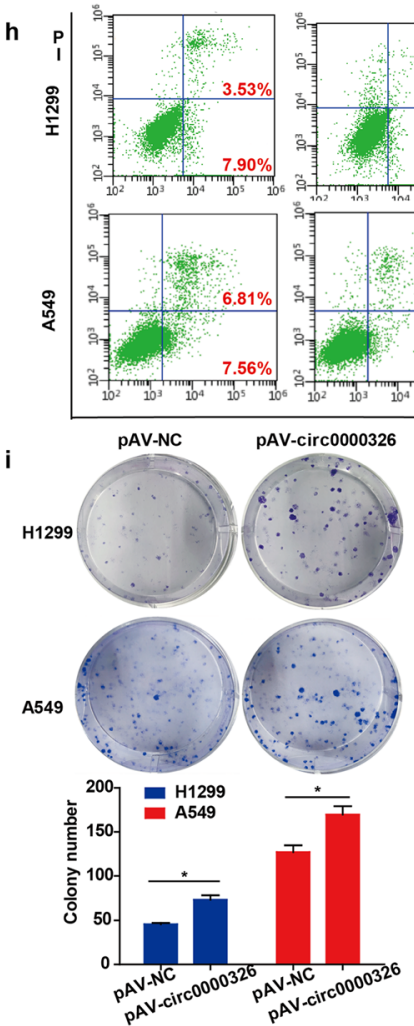
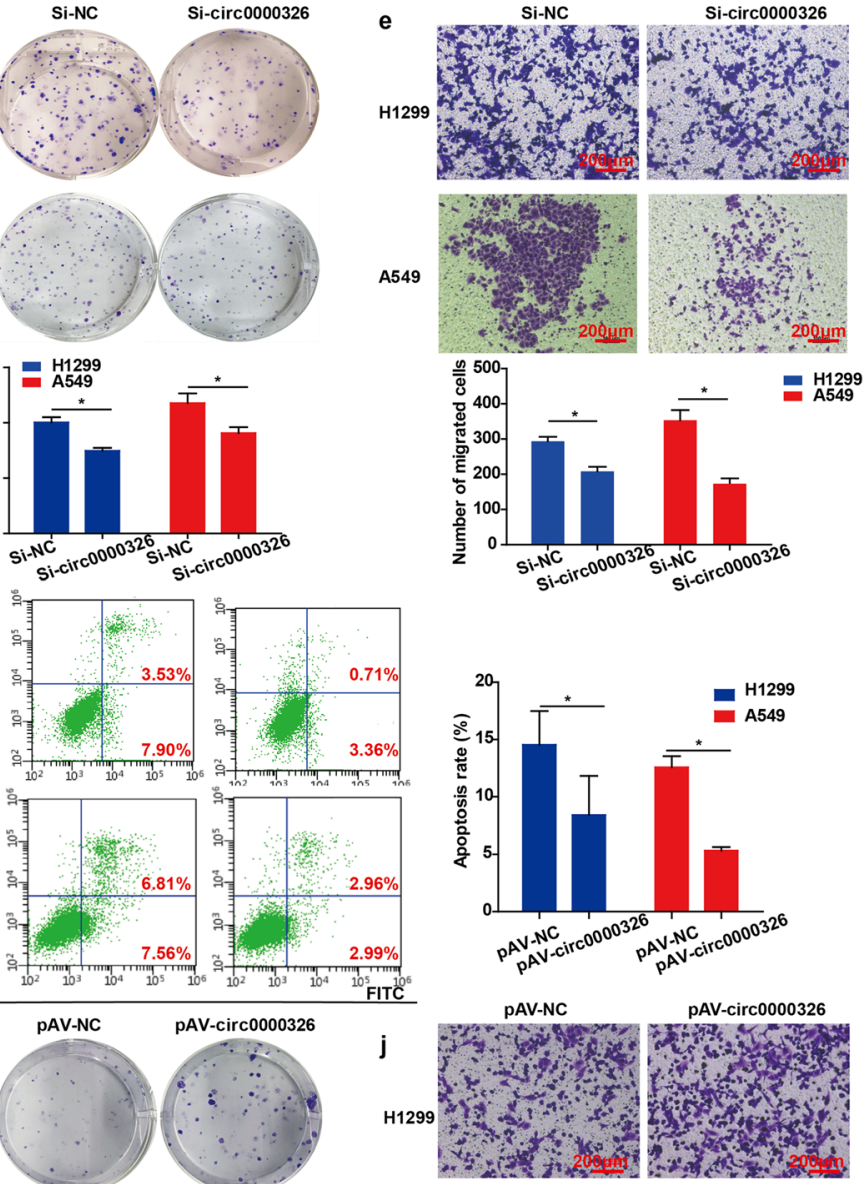

A549
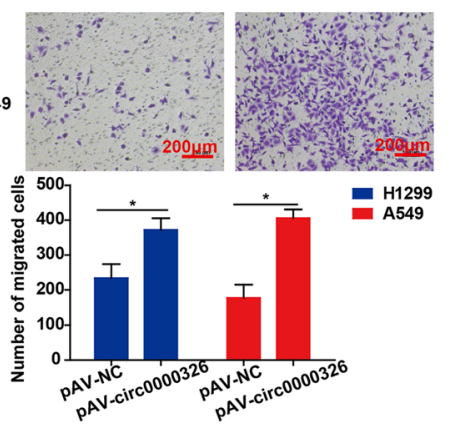

Fig. 2 (See legend on next page.) 
(See figure on previous page.)

Fig. 2 Effects of hsa_circ_0000326 expression on proliferation, apoptosis and migration in lung adenocarcinoma cells. (a) Hsa_circ_0000326 expression levels were determined by RT-PCR. (b) Effect of hsa_circ_0000326 knockdown on cell proliferation, as determined by CCK-8 assay. (c) Effect of hsa_circ_0000326 knockdown on cell apoptosis. (d) Effect of hsa_circ_0000326 knockdown on cell proliferation, as determined by colony formation assay. (e) Effect of hsa_circ_0000326 knockdown on cell migration. (f) Hsa_circ_0000326 expression levels were determined by RT-PCR. (g) Effect of hsa_circ_0000326 overexpression on cell proliferation, as determined by CCK-8 assay. (h) Effect of hsa_circ_0000326 overexpression on apoptosis. (i) Effect of hsa_circ_0000326 overexpression on cell proliferation, as determined by colony formation assay. (j) Effect of hsa_circ_0000326 overexpression on cell migration. Magnification: 100x. Scale bar $=200 \mu \mathrm{m}$. The bars with error bars represent the mean \pm SD from three independent experiments. ${ }^{*} P<0.05$

H1299 cells (Fig. 2b), while overexpression of hsa_circ_ 0000326 significantly increased the proliferation of A549, and a similar trend was also observed in H1299 cells (Fig. 2g). Similarly, based on the results of a colony formation assay, hsa_circ_0000326 knockdown significantly inhibited colony formation (Fig. 2d), while hsa

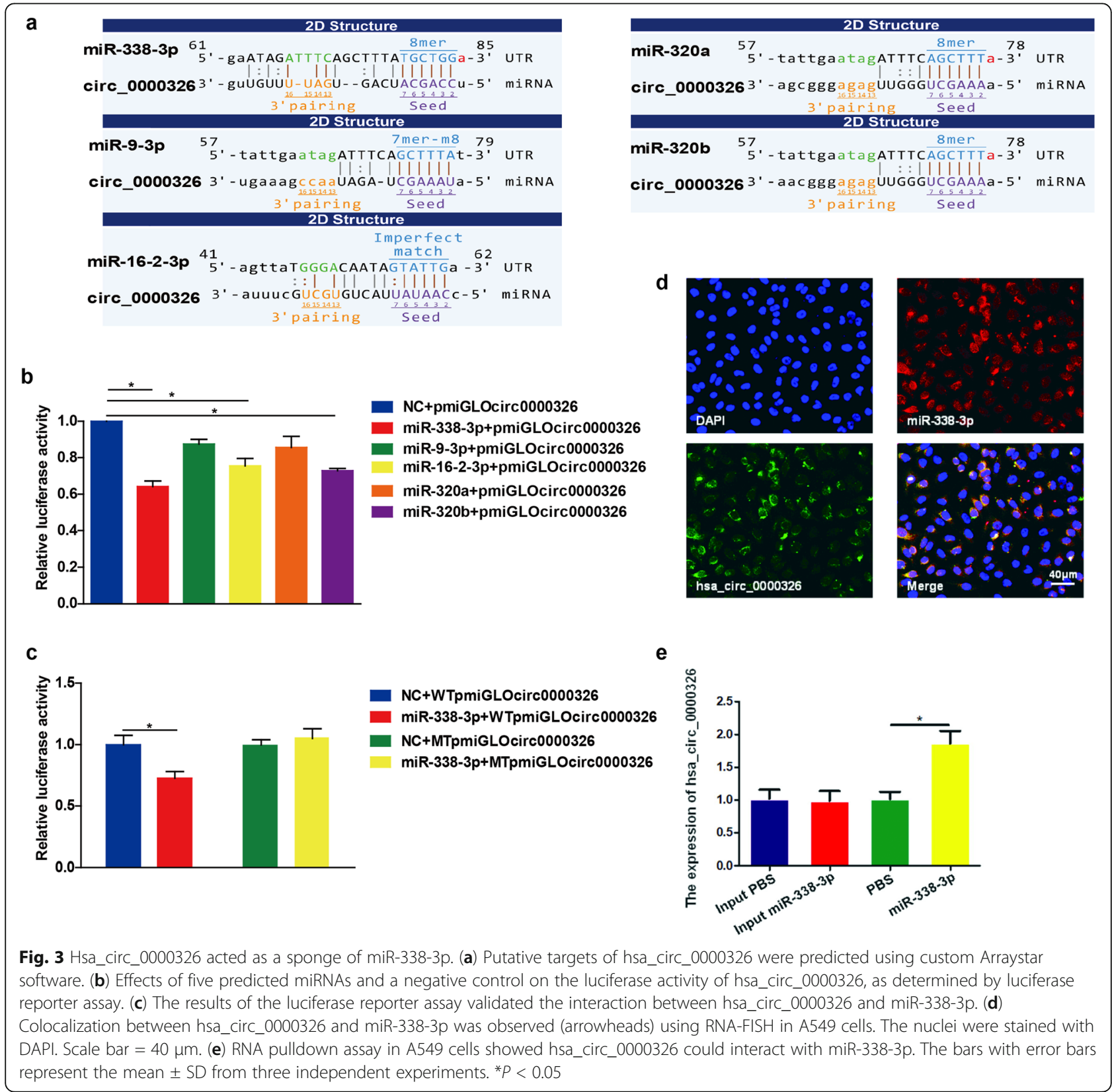




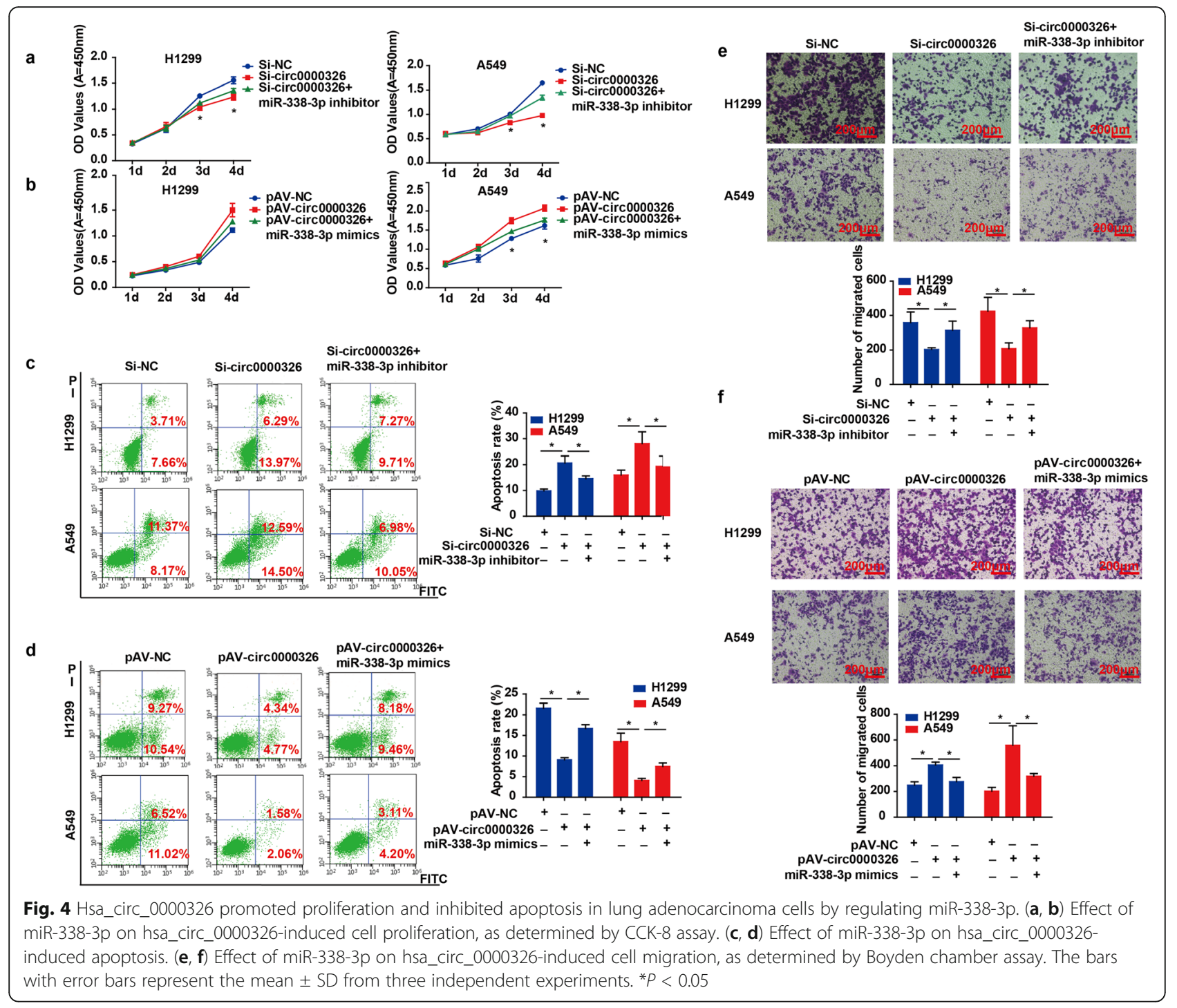

circ_0000326 overexpression promoted colony formation (Fig. 2i). Flow cytometry analysis was used to investigate the effects of hsa_circ_0000326 on the cell cycle and apoptosis and to explore the possible effect of hsa_circ 0000326 on proliferation. Cells transfected with sicirc0000326 or an overexpression plasmid showed no significant changes in the percentages of cells in the G0/G1, S, or G2/M phases (Supplement Fig. 1A-B). However, apoptosis analysis revealed knockdown of hsa_circ_0000326 expression promoted apoptosis in A549 and H1299 cells (Fig. 2c), while significantly lower apoptosis rates were observed in hsa_circ_0000326-overexpressing cells (Fig. 2h). Thus, hsa_circ_0000326 facilitated lung adenocarcinoma cell proliferation partially by inhibiting apoptosis rather than by affecting the cell cycle.

To identify the role of hsa_circ_0000326 in lung adenocarcinoma metastasis, transwell insert chambers were used to investigate the impact of hsa_circ
0000326 on cell migration, as measured by crystal violet staining. Compared to the control treatment, silencing of hsa_circ_0000326 suppressed the migration of A549 and H1299 cells across the transwell membrane (Fig. 2e). Conversely, hsa_circ_0000326 overexpression prominently increased A549 and H1299 cell migration (Fig. 2j).

Collectively, the data revealed that hsa_circ_0000326 enhanced proliferation and migration and inhibited apoptosis in lung adenocarcinoma cells.

\section{Hsa_circ_0000326 functioned as a miR-338-3p sponge and interacted with miR-338-3p}

Based on accumulating evidence, circRNAs possess numerous binding sites for miRNAs and can function as miRNA sponges to inhibit the activation of miRNAs; such sponging is one of the most common functions of circRNAs [22-25]. Thus, we hypothesized that hsa_circ_ 


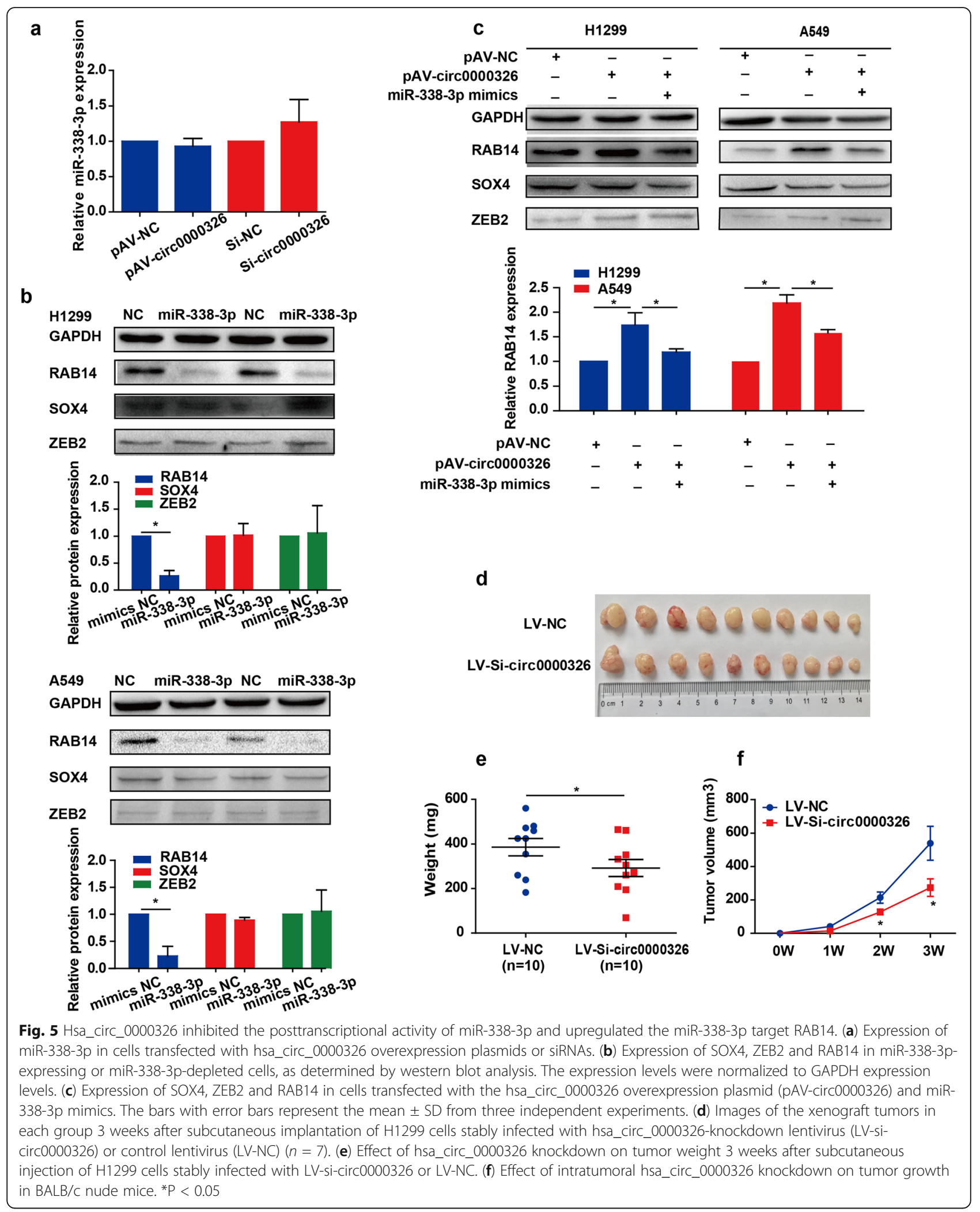


0000326 might serve as a miRNA sponge. A bioinformatics database was used to screen for miRNAs that might contain binding sites for hsa_circ_0000326. We filtered the results by condition, and five target miRNAs (miR-338-3p, miR-9-3p, miR-16-2-3p, miR-320a, and miR-320b) were chosen for further validation (Fig. 3a). To assess whether hsa_circ_0000326 could indeed bind to the predicted miRNAs, a dual luciferase reporter assay was performed. Luciferase-hsa_circ_0000326 reporters were cotransfected with miRNA mimics or negative controls, and the results indicated that compared with miRNA-NC, miR-338-3p reduced luciferase activity by $36 \%$ (Fig. 3b). Next, WTpmirGLOcirc0000326 or MTpmirGLOcirc0000326 plasmids were transiently cotransfected into cells with miR-338-3p mimics; luciferase activity was markedly lower in the wild-type hsa_circ 0000326 vector group than in the mutant vector group (Fig. 3c). Therefore, hsa_circ_0000326 interacted with miR-338-3p. Additionally, the subcellular localization of hsa_circ_0000326 and miR-338-3p was examined using RNA-FISH. Notably, hsa_circ_0000326 was mainly localized in the cytoplasm of A549 cells, consistent with the localization of miR-338-3p (Fig. 3d). In the figure, the merged picture shows the colocalization of hsa_circ 0000326 and miR-338-3p. Furthermore, RNA pulldown assay was conducted and showed the similar result (Fig. 3e). Based on these data, hsa_circ_0000326 could directly bind with miR-338-3p in the cytoplasm.

The effects of hsa_circ_0000326 on lung adenocarcinoma cells depended on miR-338-3p

We next examined whether miR-338-3p was responsible for lung adenocarcinoma cell behaviors induced by hsa circ_0000326 in vitro. We introduced miR-338-3p mimics into hsa_circ_0000326-overexpressing cells and miR-338-3p inhibitors into hsa_circ_0000326-knockdown cells. According to the results of CCK- 8 assays, silencing of hsa_circ_0000326 expression reduced the proliferation of A549 and H1299 cells, but miR-338-3p silencing attenuated this effect (Fig. 4a). Conversely, hsa circ_0000326 significantly increased the proliferation of tumor cells, but miR-338-3p mimics reversed this effect (Fig. 4b). Additionally, reintroduction of miR-338-3p inhibitors attenuated the increase in apoptosis in hsa_circ 0000326-knockdown cells (Fig. 4c). Hsa_circ_0000326 overexpression decreased the apoptosis rate, and miR338-3p mimics reversed this effect (Fig. 4d). The results of the transwell assays revealed that miR-338-3p silencing abolished the hsa_circ_0000326 knockdown-induced suppression of migration (Fig. 4e). However, hsa_circ 0000326 accelerated the migration of cells, while miR338-3p clearly reversed this effect (Fig. 4f). Thus, hsa circ_0000326 interacted with miR-338-3p to promote proliferation and migration and inhibit apoptosis in lung adenocarcinoma cells.

\section{Hsa_circ_0000326 inhibited the posttranscriptional activity of miR-338-3p and upregulated the expression of the miR-338-3p target RAB14}

According to previous studies, circRNAs sponge miRNAs to terminate their regulatory effects on target genes [26]. Interestingly, hsa_circ_0000326 did not affect the expression of miR-338-3p (Fig. 5a), indicating that hsa circ_0000326 regulated miR-338-3p at the posttranscriptional level. Because miRNAs might exert their tumorsuppressing or oncogenic functions by regulating the expression of target genes, three commonly used bioinformatics tools were applied to predict target genes. After considering the overlap among the genes predicted by TargetScan, PicTar and miRanda, RAB14, SOX4 and ZEB2 were selected as potential targets for further validation. The western blot results showed that introduction of exogenous miR-338-3p into A549 and H1299 cells reduced the levels of the RAB14 protein, indicating that RAB14, but not SOX4 or ZEB2, was the downstream target of miR-338-3p (Fig. 5b). Next, we explored whether hsa_circ_0000326 regulated the expression of RAB14. As shown in the western blots, hsa_circ 0000326 overexpression dramatically increased the levels of the RAB14 protein, and reintroduction of miR-338-3p reversed this effect (Fig. 5c).

Silencing of hsa_circ_0000326 suppressed tumor growth H1299 cells that were stably infected with LV-sicirc0000326 or LV-NC were subcutaneously injected into the right armpits of nude mice. Three weeks after injection, the tumors were removed and photographed (Fig. 5d). The weights of the xenograft tumors in which hsa_circ_0000326 expression was downregulated were significantly lower than those of the control xenograft tumors (Fig. 5e). In addition, tumors derived from circ0000326-knockdown clones grew at significantly slower rates than control tumors at all time points examined (Fig. 5f).

\section{Discussion}

According to recent studies, circRNAs represent potentially novel and promising biomarkers in cancer and other diseases, such as hsa_circ_0000190 in gastric cancer [27], hsa_circRNA_103636 in major depressive disorder [28], and hsa_circ_0004277 in acute myeloid leukemia [29]. Functional and mechanistic studies have revealed that circRNAs exert regulatory effects on the initiation and progression of diseases [30, 31]. Notably, circRNAs have been shown to act as competing endogenous RNAs via the circRNA-miRNA-mRNA signaling axis, which participates in the initiation and 
progression of various cancers, such as colorectal cancer [32], gastric cancer [33], breast cancer [34], and esophageal cell carcinoma $[35,36]$.

Based on accumulating evidence, aberrant circRNA expression plays critical roles in lung cancer. Liu et al. reported the upregulation of certain circRNAs
(circ-ZEB1.5, circ-ZEB1.19, circ-ZEB1.17 and circZEB1.33) in normal lung tissue samples compared to lung cancer samples [37]. Yao et al. observed elevated expression of circRNA_100876 in NSCLC tissues compared to adjacent lung tissues by RT-PCR. Furthermore, Kaplan-Meier survival analysis showed that

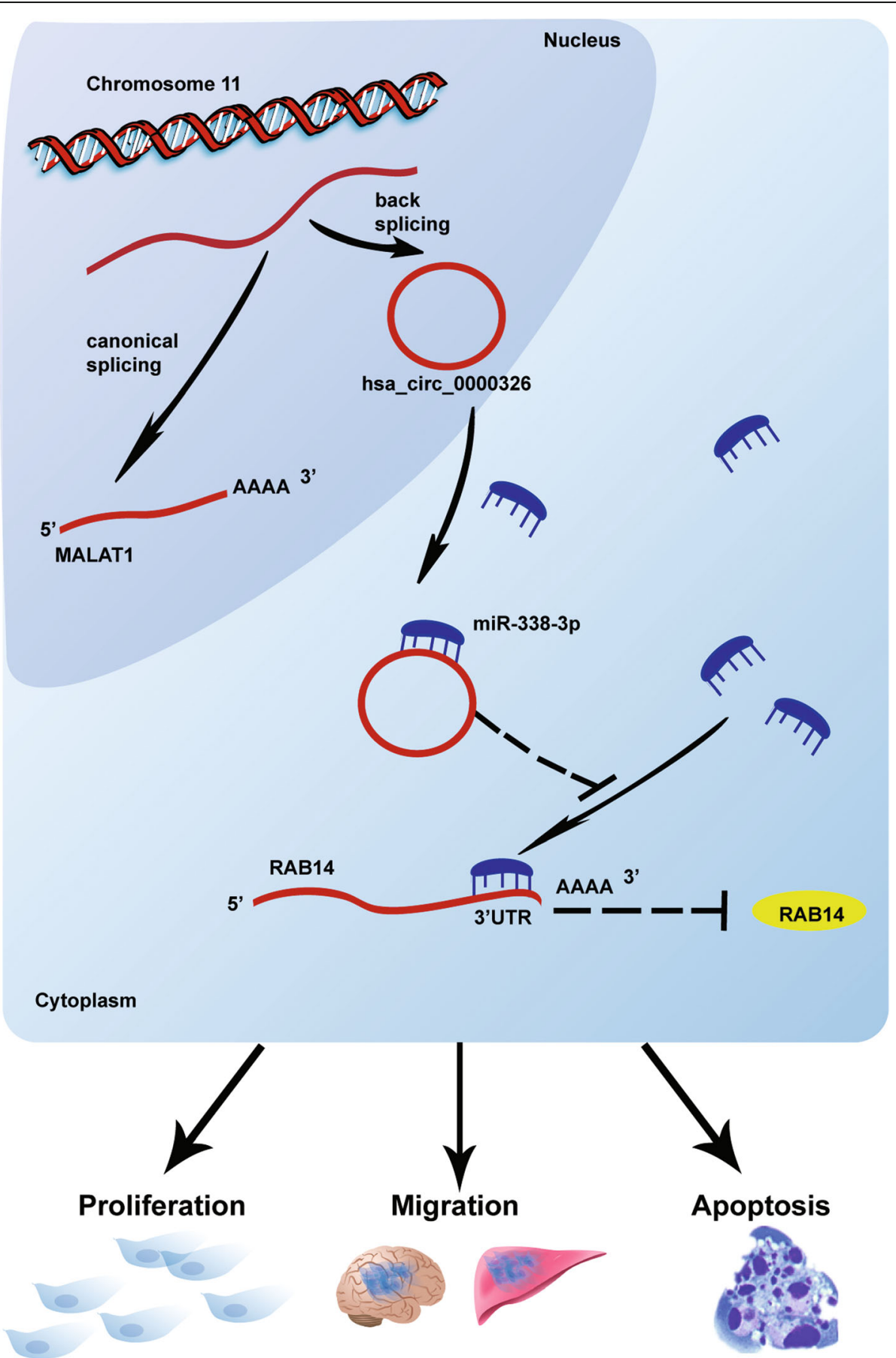

Fig. 6 Schematic of the biological roles of hsa_circ_0000326 in lung adenocarcinoma carcinogenesis. Hsa_circ_0000326 could bind to miR-338$3 p$ as a miRNA sponge, exerting its function via regulation of the downstream target RAB14. Knockdown of hsa_circ_0000326 could inhibit lung adenocarcinoma cell proliferation and migration and promote apoptosis in vitro and inhibit tumor formation in vivo 
patients expressing high levels of circRNA_100876 had reduced overall survival times, and the levels of circRNA_100876 expression were found to be correlated with lymph node metastasis and NSCLC tumor stage [38]. Using microarray screening, Zhu et al. revealed that hsa_circ_0013958 expression is markedly upregulated in lung adenocarcinoma tissues. Hsa circ_0013958 promotes cell proliferation and invasion and inhibits apoptosis by acting as a sponge of miR134 and thus upregulating the expression of oncogenic cyclin D1 [39]. The classic circRNA ITCH has been found to be significantly downregulated in lung cancer tissues and to function as a sponge for miR-7 and miR-214, subsequently suppressing the activity of the $\mathrm{Wnt} / \beta$-catenin signaling pathway [40]. According to microarray and RT-PCR data, hsa_circ_0043256 is upregulated in NSCLC cells in response to cinnamaldehyde treatment and might inhibit cell proliferation and induce apoptosis via the hsa_circ_0043256/miR$1252 / \mathrm{ITCH}$ axis in response to cinnamaldehyde [41].

In the present study, we used microarray technology to show that hsa_circ_0000326 expression was significantly upregulated in lung adenocarcinoma tissues, and we validated this result with a larger sample size using RT-PCR. Moreover, we found that high hsa_circ 0000326 expression correlated with $\mathrm{T}$ stage, $\mathrm{N}$ stage and tumor differentiation level. We then explored the biological role of hsa_circ_0000326 using gain- and loss-offunction assays. Hsa_circ_0000326 promoted cell proliferation by inhibiting apoptosis rather than by affecting the cell cycle and increased the migration of lung adenocarcinoma cells.

Based on emerging data, circRNAs exert key regulatory effects by acting as competing endogenous RNAs. According to bioinformatics analysis, miR-338-3p was predicted to interact with hsa_circ_0000326, and luciferase reporter assays further validated this interaction. We detected the subcellular localization of hsa_circ_0000326 and found that hsa_circ_0000326 was mainly located in the cytoplasm, the same subcellular location as miR338-3p. Functional assays showed that miR-338-3p mimics reversed the effects of hsa_circ_0000326 overexpression and that miR-338-3p inhibition reversed the effects of hsa_circ_0000326 depletion. However, hsa_circ_ 0000326 did not affect miR-338-3p expression. In our opinion, hsa_circ_0000326 may competitively bind and inhibit the activity of miR-338-3p, rather than effect the expression. Just as previous study, CircNT5E [26] or hsa_circ_001569 [42] or Circular RNA-ZNF609 [43] or circRHOBTB3 [44] were identified as a sponge of miRNA and did not affect their expression. Therefore, by acting as a miR-338-3p sponge, hsa_circ_0000326 may competitively bind to and inhibit miR-338-3p activity, resulting in increased expression of miR-338-3p targets.

As shown in previous studies, miR-338-3p expression exhibits marked changes in various tumors, such as hepatocellular carcinoma [45], gastric cancer [46], and lung cancer [47]. In the present study, hsa_circ_0000326 functioned at the posttranscriptional level to inhibit the activity of miR-338-3p and prominently increased the proliferation and migration of lung adenocarcinoma cancer cells by acting as a miR-338-3p sponge. Consistent with the results of previous studies, miR-338-3p expression was significantly decreased and functioned as a tumor suppressor. Importantly, miR-338-3p inhibits proliferation and the epithelial-mesenchymal transition in vitro and suppresses tumor growth in vivo $[48,49]$.

Using three bioinformatic algorithms (TargetScan, PicTar and miRanda), we identified RAB14, SOX4 and ZEB2 as potential targets of miR-338-3p. RAB14 is a member of the RAB family of low-molecular-weight GTPases and is expressed in a wide range of cell lines [50]. As shown in the western blots, RAB14 was a target of miR-338-3p in lung adenocarcinoma cells, and hsa circ_0000326 overexpression contributed to elevated levels of RAB14. Alterations in RAB14 expression have been documented in gastric cancer, renal cancer, nasopharyngeal carcinoma and lung cancer $[47,51,52]$. As an oncogene, RAB14 participates in many biological processes, such as cell proliferation, migration and invasion, by regulating the AKT signaling pathway [51, 53].

Finally, we established xenografts in nude mice to assess the function of hsa_circ_0000326 in vivo. Interestingly, hsa_circ_0000326 affected lung adenocarcinoma progression in vivo, as evidenced by the significantly lower weights and volumes LV-si-circ0000326 xenograft tumors than control xenograft tumors.

Our study had some limitations. First, due to the poor tumorigenesis of A549 cells in nude mice, we did not create stable knockdown hsa_circ_0000326 cell lines or subcutaneously inject mice with these cells. Second, the role of hsa_circ_0000326 on the tumor cell invasion was unknown, although we have conducted invasion assay and optimized the experimental conditions for many times. Third, A549 and H1299 were used as the experimental cells because of the significantly high expression of hsa_circ_0000326. Although the different expression pattern of P53 in A549 and H1299 cells and P53 acted as a tumor suppressor, we observed that hsa_circ 0000326 acted as a negative regulator of apoptosis, along with RAB14 overexpression in A549 and H1299 cells, which indicated P53 may not be the target for hsa_circ 0000326/miR-338-3p/RAB14 signal pathway. A recently study found RAB14 overexpression upregulated $\mathrm{Bcl}-2$, an important anti-apoptotic protein thus inhibited apoptosis [54]. We hypothesis the underlying apoptosis 
mechanisms of hsa_circ_0000326 may be upregulated of $\mathrm{Bcl}-2$ expression, which need to be further validated.

Aberrant hsa_circ_0000326 expression in lung adenocarcinoma promotes tumor proliferation and metastasis. We have constructed a model of the mechanism of hsa circ_0000326 (Fig. 6) showing that the covalent binding of hsa_circ_0000326 to miR-338-3p increases RAB14 expression and participates in the progression of lung adenocarcinoma.

\section{Conclusions}

Our study results reveal crucial roles for hsa_circ 0000326 in the proliferation, migration and apoptosis of lung adenocarcinoma cells and suggest that hsa_circ 0000326 may represent a potential therapeutic target in patients with lung adenocarcinoma.

\section{Supplementary information}

Supplementary information accompanies this paper at https://doi.org/10 1186/s13046-020-01556-4.

\section{Additional file 1: Supplement fig. 1. The expression of}

hsa_circ_0000326 had no effect on the cell cycle of A549. (a) Effect of

hsa_circ_0000326 knockdown on cell cycle. (b) Effect of

hsa_circ_0000326 overexpression on cell cycle, as determined by colony formation assay.

Additional file 2: Supplement fig. 2. RNAase $H$ digestion results for hsa_circ_0000326.

Additional file 3: Table S1. The results of circRNAs array for lung adenocarcinoma tissues and adjacent normal tissues.

Additional file 4: Table S2. Dysregulated circRNAs in lung adenocarcinoma tissues compared with adjacent normal tissues.

Additional file 5: Table S3. Correlation between Circ_0000326 expression and clinical pathological characteristics.

\section{Abbreviations}

circRNAs: Circular RNAs; NSCLCs: Non-small cell lung cancers; FISH: Fluorescence in situ hybridization; MALAT1: Metastasis associated lung adenocarcinoma transcript 1; SD: Standard deviation; RAB14: Ras-related protein Rab-14; SOX4: SRY-Box Transcription Factor 4; ZEB2: Zinc Finger E-Box Binding Homeobox 2

\section{Acknowledgements}

We appreciate Nature Research Editing Service for language editing for us.

\section{Authors' contributions}

WNX and JPZ designed, edited and led out the experiments of this study. $Y Z X, J Y, Z L H, Y T, X F Q, Y M$ and $Y N H$ conducted the experiments, data analysis, and critical discussions of the results. YW, YC, DSJ, JGX and YJX provided material support and study supervision. All authors contributed to this manuscript. All authors read and approved the final manuscript.

\section{Funding}

This work was supported by funding from the National Natural Science Foundation of China (81500055). Wuhan Young and Middle-aged Medical Key Talents Training Project. Clinical Research Physician Program of Tongji Medical College, Integrated Innovative Team for Major Human Diseases Program of Tongji Medical College, HUST.

\section{Availability of data and materials}

The datasets used and/or analysed during the current study are available from the corresponding author on reasonable request.

\section{Ethics approval and consent to participate}

Human lung adenocarcinoma tissues and corresponding adjacent tissues was obtained from the Tongji Hospital under the approval by the ethics committee of Tongji Hospital Ethics Review Board. All the animal experiments performed in this study were approved by the Institutional Animal Care and Use Committee of Tongji Hospital, Tongji Medical College, Huazhong University of Science and Technology.

\section{Consent for publication}

Not applicable.

\section{Competing interests}

The authors declare that they have no competing interests.

\section{Author details}

'Department of Respiratory and Critical Care Medicine, Wuhan Clinical Medical Research Center for Chronic Airway Medicine, NHC Key Laboratory of Pulmonary Diseases, Key cite of National Clinical Research Center for Respiratory Diseases, Tongji Hospital, Tongji Medical College, Huazhong University of Sciences \& Technology, 1095 Jiefang Ave, Wuhan 430030, China. ${ }^{2}$ Department of Respiratory, Zhuzhou Central Hospital, Zhuzhou, China. ${ }^{3}$ Department of Thoracic Surgery, Tongji Hospital, Tongji Medical College, Huazhong University of Science and Technology, Wuhan, China. ${ }^{4}$ Division of Cardiothoracic and Vascular Surgery, Tongji Hospital, Tongji Medical College, Huazhong University of Science and Technology, Wuhan, China. ${ }^{5}$ Department of Respiratory Medicine, Shanghai Ninth People's Hospital, Shanghai Jiaotong University School of Medicine, 639 Zhizaoju Lu, Shanghai 200011, China

Received: 18 September 2019 Accepted: 8 March 2020

Published online: 05 April 2020

\section{References}

1. Smith RA, Manassaram-Baptiste D, Brooks D, Doroshenk M, Fedewa S, Saslow D, et al. Cancer screening in the United States, 2015: a review of current American cancer society guidelines and current issues in cancer screening. CA Cancer J Clin. 2015:65:30-54.

2. Ettinger DS, Wood DE, Akerley W, Bazhenova LA, Borghaei H, Camidge DR, et al. Non-small cell lung cancer, version 6.2015. J National Comprehensive Cancer Network. 2015;13:515-24

3. Nicholson AG, Chansky K, Crowley J, Beyruti R, Kubota K, Turrisi A, et al. The International Association for the Study of Lung Cancer lung Cancer staging project: proposals for the revision of the clinical and pathologic staging of small cell lung cancer in the forthcoming eighth edition of the TNM classification for lung cancer. J Thoracic Oncol. 2016;11:300-11.

4. Salzman J, Gawad C, Wang PL, Lacayo N, Brown PO. Circular RNAs are the predominant transcript isoform from hundreds of human genes in diverse cell types. PLoS One. 2012;7:e30733.

5. Kristensen LS, Hansen TB, Veno MT, Kjems J. Circular RNAs in cancer: opportunities and challenges in the field. Oncogene. 2018;37:555-65.

6. Cocquerelle C, Mascrez B, Hetuin D, Bailleul B. Mis-splicing yields circular RNA molecules. FASEB J. 1993;7:155-60.

7. Jeck WR, Sorrentino JA, Wang K, Slevin MK, Burd CE, Liu J, et al. Circular RNAs are abundant, conserved, and associated with ALU repeats. RNA (New York, N.Y.). 2013;19:141-57.

8. Wang S, Zhang X, Li Z, Wang W, Li B, Huang X, et al. Circular RNA profile identifies circOSBPL10 as an oncogenic factor and prognostic marker in gastric cancer. Oncogene. 2019;38:6985-7001.

9. Memczak S, Jens M, Elefsinioti A, Torti F, Krueger J, Rybak A, et al. Circular RNAs are a large class of animal RNAs with regulatory potency. Nature. 2013:495:333-8.

10. Hansen TB, Jensen TI, Clausen BH, Bramsen JB, Finsen B, Damgaard CK, et al. Natural RNA circles function as efficient microRNA sponges. Nature. 2013; 495:384-8.

11. Hansen TB, Wiklund ED, Bramsen JB, Villadsen SB, Statham AL, Clark SJ, et al. miRNA-dependent gene silencing involving Ago2-mediated cleavage of a circular antisense RNA. EMBO J. 2011;30:4414-22.

12. Ashwal-Fluss R, Meyer M, Pamudurti NR, Ivanov A, Bartok O, Hanan M, et al. circRNA biogenesis competes with pre-mRNA splicing. Mol Cell. 2014;56:55-66.

13. Rayner R, Carville K, Leslie G, Roberts P. A review of patient and skin characteristics associated with skin tears. J Wound Care. 2015;24:406-14. 
14. Wang Y, Wang Z. Efficient backsplicing produces translatable circular mRNAs. RNA (New York, N.Y.). 2015;21:172-9.

15. Cheng Z, Yu C, Cui S, Wang H, Jin H, Wang C, et al. circTP63 functions as a ceRNA to promote lung squamous cell carcinoma progression by upregulating FOXM1. Nature Communications. 2019;10:3200.

16. Wang L, Liang Y, Mao Q, Xia W, Chen B, Shen $\mathrm{H}$, et al. Circular RNA circCRIM1 inhibits invasion and metastasis in lung adenocarcinoma through the microRNA (miR)-182/miR-93-leukemia inhibitory factor receptor pathway. Cancer Sci. 2019;110:2960-72.

17. Wang Y, Zhu J, Zhang L, Zhang Z, He L, Mou Y, et al. Role of C/EBP homologous protein and endoplasmic reticulum stress in asthma exacerbation by regulating the IL-4/signal transducer and activator of transcription 6/transcription factor EC/IL-4 receptor alpha positive feedback loop in M2 macrophages. J Allergy Clin Immunol. 2017;140:1550-61 e1558.

18. Qi X, Chen H, Huang Z, Fu B, Wang Y, Xie J, et al. Aberrantly expressed IncRNAs identified by microarray analysis in CD4(+)T cells in asthmatic patients. Biochem Biophys Res Commun. 2018;503:1557-62.

19. Yao Y, Wang Y, Zhang Z, He L, Zhu J, Zhang M, et al. Chop deficiency protects mice against Bleomycin-induced pulmonary fibrosis by attenuating M2 macrophage production. Mol Ther. 2016;24:915-25.

20. Chen $H, X u X$, Cheng $S, X u Y$, Xuefei $Q$, Cao $Y$, et al. Small interfering RNA directed against microRNA-155 delivered by a lentiviral vector attenuates asthmatic features in a mouse model of allergic asthma. Exper Therapeutic Med. 2017;14:4391-6.

21. Lu Q, Liu T, Feng H, Yang R, Zhao X, Chen W, et al. Circular RNA circSLC8A1 acts as a sponge of miR-130b/miR-494 in suppressing bladder cancer progression via regulating PTEN. Mol Cancer. 2019;18:111.

22. Hansen TB, Kjems J, Damgaard CK. Circular RNA and miR-7 in cancer. Cancer Res. 2013;73:5609-12.

23. Zhao ZJ, Shen J. Circular RNA participates in the carcinogenesis and the malignant behavior of cancer. RNA Biol. 2017;14:514-21.

24. Dong Y, He D, Peng Z, Peng W, Shi W, Wang J, et al. Circular RNAs in cancer: an emerging key player. J Hematol Oncol. 2017;10:2.

25. Meng S, Zhou H, Feng Z, Xu Z, Tang Y, Li P, et al. CircRNA: functions and properties of a novel potential biomarker for cancer. Mol Cancer. 2017;16:94.

26. Wang R, Zhang S, Chen X, Li N, Li J, Jia R, et al. CircNT5E acts as a sponge of miR422a to promote Glioblastoma tumorigenesis. Cancer Res. 2018;78:4812-25.

27. Chen S, Li T, Zhao Q, Xiao B, Guo J. Using circular RNA hsa_circ_0000190 as a new biomarker in the diagnosis of gastric cancer. Clinica Chimica Acta. 2017:466:167-71.

28. Cui X, Niu W, Kong L, He M, Jiang K, Chen S, et al. hsa_circRNA_103636: potential novel diagnostic and therapeutic biomarker in major depressive disorder. Biomark Med. 2016;10:943-52.

29. Li W, Zhong C, Jiao J, Li P, Cui B, Ji C, et al. Characterization of hsa circ 0004277 as a new biomarker for acute myeloid leukemia via circular RNA profile and bioinformatics analysis. Int J Mol Sci. 2017;18:597.

30. Chen J, Wang J, Jiang Y, Gu W, Ni B, Sun H, et al. Identification of circular RNAs in human aortic valves. Gene. 2018:642:135-44.

31. Zhuang ZG, Zhang JA, Luo HL, Liu GB, Lu YB, Ge NH, et al. The circular RNA of peripheral blood mononuclear cells: Hsa_circ_0005836 as a new diagnostic biomarker and therapeutic target of active pulmonary tuberculosis. Mol Immunol. 2017;90:264-72.

32. Hsiao KY, Lin YC, Gupta SK, Chang N, Yen L, Sun HS, et al. Noncoding effects of circular RNA CCDC66 promote Colon Cancer growth and metastasis. Cancer Res. 2017;77:2339-50.

33. Shao $Y$, Chen $L$, Lu R, Zhang $X$, Xiao B, Ye G, et al. Decreased expression of hsa_circ_0001895 in human gastric cancer and its clinical significances. Tumour Biol. 2017;39:1010428317699125.

34. Liang G, Liu Z, Tan L, Su AN, Jiang WG, Gong C. HIF1alpha-associated circDENND4C promotes proliferation of breast Cancer cells in hypoxic environment. Anticancer Res. 2017;37:4337-43.

35. Xia W, Qiu M, Chen R, Wang S, Leng X, Wang J, et al. Circular RNA has_circ_ 0067934 is upregulated in esophageal squamous cell carcinoma and promoted proliferation. Sci Rep. 2016;6:35576.

36. Li F, Zhang L, Li W, Deng J, Zheng J, An M, et al. Circular RNA ITCH has inhibitory effect on ESCC by suppressing the Wnt/beta-catenin pathway. Oncotarget. 2015;6:6001-13.

37. Liu YC, Li JR, Sun CH, Andrews E, Chao RF, Lin FM, et al. CircNet: a database of circular RNAs derived from transcriptome sequencing data. Nucleic Acids Res. 2016:44:D209-15.
38. Yao JT, Zhao SH, Liu QP, Lv MQ, Zhou DX, Liao ZJ, et al. Over-expression of CircRNA_100876 in non-small cell lung cancer and its prognostic value. Pathol Res Pract. 2017;213:453-6.

39. Zhu X, Wang X, Wei S, Chen Y, Chen Y, Fan X, et al. hsa circ 0013958: a circular RNA and potential novel biomarker for lung adenocarcinoma. FEBS J. 2017;284:2170-82.

40. Wan L, Zhang L, Fan K, Cheng ZX, Sun QC, Wang JJ. Circular RNA-ITCH suppresses lung Cancer proliferation via inhibiting the Wnt/beta-catenin pathway. Biomed Res Int. 2016;2016:1579490.

41. Tian F, Yu CT, Ye WD, Wang Q. Cinnamaldehyde induces cell apoptosis mediated by a novel circular RNA hsa_circ_0043256 in non-small cell lung cancer. Biochem Biophys Res Commun. 2017;493:1260-6.

42. Xie H, Ren X, Xin S, Lan X, Lu G, Lin Y, et al. Emerging roles of circRNA 001569 targeting miR-145 in the proliferation and invasion of colorectal cancer. Oncotarget. 2016;7:26680-91.

43. Wu P, Zhang D, Geng Y, Li R, Zhang Y. Circular RNA-ZNF609 regulates corneal neovascularization by acting as a sponge of miR-184. Exp Eye Res. 2020;192:107937.

44. Deng G, Mou T, He J, Chen D, Lv D, Liu H, et al. Circular RNA circRHOBTB3 acts as a sponge for miR-654-3p inhibiting gastric cancer growth. J Exper Clin Cancer Res. 2020;39:1.

45. Zhang T, Liu W, Zeng XC, Jiang N, Fu BS, Guo Y, et al. Down-regulation of microRNA-338-3p promoted angiogenesis in hepatocellular carcinoma. Biomed Pharmacother. 2016;84:583-91.

46. Huang $N$, Wu Z, Lin L, Zhou M, Wang L, Ma H, et al. MiR-338-3p inhibits epithelial-mesenchymal transition in gastric cancer cells by targeting ZEB2 and MACC1/met/Akt signaling. Oncotarget. 2015;6:15222-34.

47. Sun J, Feng X, Gao S, Xiao Z. microRNA-338-3p functions as a tumor suppressor in human nonsmallcell lung carcinoma and targets Ras-related protein 14. Mol Med Reports. 2015;11:1400-6.

48. Guo B, Liu L, Yao J, Ma R, Chang D, Li Z, et al. miR-338-3p suppresses gastric cancer progression through a PTEN-AKT axis by targeting P-REX2a. Mol Cancer Res. 2014;12:313-21.

49. Shan Y, Li X, You B, Shi S, Zhang Q, You Y. MicroRNA-338 inhibits migration and proliferation by targeting hypoxia-induced factor 1alpha in nasopharyngeal carcinoma. Oncol Rep. 2015;34:1943-52.

50. Taglauer ES, Artemiuk PA, Hanscom SR, Lindsay AJ, Wuebbolt D, Breathnach FM, et al. Rab11 family expression in the human placenta: localization at the maternal-fetal interface. PLoS One. 2017;12:e0184864

51. Guo B, Wang W, Zhao Z, Li Q, Zhou K, Zhao L, et al. Rab14 act as oncogene and induce proliferation of gastric Cancer cells via AKT signaling pathway. PLoS One. 2017;12:e0170620.

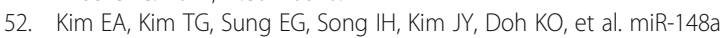
increases the sensitivity to cisplatin by targeting Rab14 in renal cancer cells. Int J Oncol. 2017;50:984-92.

53. Linford A, Yoshimura S, Nunes BR, Langemeyer L, Gerondopoulos A, Rigden DJ, et al. Rab14 and its exchange factor FAM116 link endocytic recycling and adherens junction stability in migrating cells. Dev Cell. 2012;22:952-66.

54. Ge J, Ge C. Rab14 overexpression regulates gemcitabine sensitivity through regulation of $\mathrm{BCl}-2$ and mitochondrial function in pancreatic cancer. Virchows Archiv. 2019;474:59-69.

\section{Publisher's Note}

Springer Nature remains neutral with regard to jurisdictional claims in published maps and institutional affiliations.

Ready to submit your research? Choose BMC and benefit from:

- fast, convenient online submission

- thorough peer review by experienced researchers in your field

- rapid publication on acceptance

- support for research data, including large and complex data types

- gold Open Access which fosters wider collaboration and increased citations

- maximum visibility for your research: over $100 \mathrm{M}$ website views per year

At BMC, research is always in progress.

Learn more biomedcentral.com/submission 